\title{
Supplier diversification under binomial yield
}

\author{
Mehmet Murat Fadıloğlu a,*, Emre Berk ${ }^{\mathrm{b}}$, Mustafa Çağrı Gürbüz ${ }^{\mathrm{c}}$ \\ a Department of Industrial Engineering, Bilkent University, Ankara, Turkey \\ b Department of Management, Bilkent University, Ankara, Turkey \\ ${ }^{\mathrm{c}}$ MIT-Zaragoza Logistics Center, Zaragoza, Spain
}

\section{A R T I C L E I N F O}

\section{Article history:}

Received 27 July 2007

Accepted 7 May 2008

Available online 20 June 2008

\section{Keywords:}

Inventory models

EOQ

Random yield

Multiple suppliers

\begin{abstract}
A B S T R A C T
We consider supplier diversification in an EOQ type inventory setting with multiple suppliers and binomial yields. We characterize the optimal policy for the model and show that, in this case, it does not pay to diversify, in contrast to previous results in the random yield literature.
\end{abstract}

(C) 2008 Elsevier B.V. All rights reserved.

\section{Introduction}

In this paper, we analyze an inventory system with possible multiple suppliers under binomial yield, characterize the optimal replenishment policy, and show that working with a single supplier is always optimal.

There is a vast literature on inventory models facing yield uncertainty. Yano and Lee [16] classify random yield models in the literature into four categories: Binomial yield, batch size independent-stochastically proportional yield, batch size dependent-stochastically proportional yield and random capacity.

Binomial yield differs from the rest in the important aspect that it exhibits independence across units in a given order whereas intra-batch correlation is implied in all other categories. Mazzola et al. [11] provides the earliest work on an inventory model with binomial yield. For other works in this category, see Beja [3], Grosfeld-Nir and Gerchak [8], Barad and Braha [2], Sepheri et al. [14], and Gürbüz [10].

For a comprehensive review of individual works in the remaining categories, we refer the reader to Yano and Lee [16], and mention only Erdem and Ozekici [5], Gerchak et al. [7], Gurnani et al. [9], Wang and Gerchak [15] and Erdem et al. [4] as recent works.

Most of the previous work has focused on inventory systems with a single supplier and does not consider diversification

\footnotetext{
* Corresponding author.

E-mail addresses: mmurat@bilkent.edu.tr (M.M. Fadıloğlu), eberk@bilkent.edu.tr (E. Berk), mgurbuz@zlc.edu.es (M.Ç. Gürbüz).
}

issues. However, in practice buyers can work with multiple suppliers, and thus may reduce variability of actual yield through diversification among suppliers. Anupindi and Akella [1], Erdem and Ozekici [5], Gerchak and Parlar [6], Parlar and Wang [13] consider diversification under different yield structures (excluding binomial yield), and Gürbüz [10] under binomial yield. Erdem et al. [4] allow for more than two suppliers in the presence of random capacity.

There is also evidence from practice that diversification is desirable for certain settings. A good example is furnished by Li \& Fung, the supply chain integrator, which connects the fragmented supplier market (Asia, Indian subcontinent, the Caribbean basin) to the large retailers in the US and Western Europe. When Li \& Fung receives an order from a retailer, say for a 1000 units, depending on the characteristic of the product and the particular supply chain, they procure the item from, say, 5 different suppliers (even within the same country), 200 from each (see [12]). However, in this paper we show that, under binomial supplier yield, working with a single supplier is always optimal. Thus, not all sources of uncertainty can be remedied through diversification.

The rest of the paper is organized as follows: In Section 2, we describe the model. Sections 3 and 4 present the optimality results and the discussion. Section 5 concludes the paper with possible extensions of this work.

\section{Model}

We consider an EOQ type inventory model in which demand occurs continuously at a constant rate $D$. The system is subject to an inventory holding cost $h$ per unit held in inventory per unit time. No backorders are allowed. The system is replenished by 
ordering from $n(n \leq N)$ suppliers where $N$ denotes the total number of available suppliers. We assume that the replenishments are instantaneous (i.e., zero lead time) and that each unit delivered by supplier $i$, where $i=1,2, \ldots, N$, has a constant probability that it is of acceptable quality, $p_{i}$, independent of the order quantity, $Q_{i}$. The system pays a unit purchasing $\operatorname{cost} c_{i}$ for each unit ordered irrespective of its quality. Later, the results are also extended to the case where the system pays only for the "good" units. The "bad" units are discarded right at the delivery (and hence do not join the stock and do not induce any holding cost). There is an ordering cost $K\left(Q_{1}, \ldots, Q_{N}\right)$, which is a positive function of the order quantities $Q_{i}$. We assume that $K\left(Q_{1}, \ldots, Q_{N}\right)$ is nondecreasing in each order quantity $Q_{i}$.

We employ the following EOQ type ordering policy: The inventory level is monitored continuously and an order of size $Q_{i}$ is placed at supplier $i$ whenever inventory level hits zero. Clearly there is no incentive to order prior to reaching zero inventory level because of the zero lead time assumption. The objective is to minimize the total long-run average cost, which consists of ordering, purchasing and holding components. All the order quantities have to be optimized simultaneously in order to characterize the optimal policy parameters.

The reader should note that the number of acceptable units from supplier $i$ is the sum of $Q_{i}$ independent Bernouilli random variables and thereby has binomial distribution with parameters $p_{i}$ and $Q_{i}$. We call this quantity the effective received from supplier $i$ and denote it as $R_{i}$. The numbers of the effectives received from different suppliers are independent. The sum of these quantities from all suppliers is the total effective received $R$ where

$R=\sum_{i=1}^{N} R_{i}$

We can easily compute the expected values of $R$ and of $R^{2}$ as

$$
\begin{aligned}
E[R] & =\sum_{i=1}^{N} E\left[R_{i}\right]=\sum_{i=1}^{N} p_{i} Q_{i} \text { and } \\
E\left[R^{2}\right] & =\sum_{i=1}^{N} E\left[R_{i}^{2}\right]+2 \sum_{i=1}^{N} \sum_{j=i+1}^{N} E\left[R_{i}\right] E\left[R_{j}\right] \\
& =\sum_{i=1}^{N} \operatorname{Var}\left(R_{i}\right)+\sum_{i=1}^{N} E^{2}\left[R_{i}\right]+2 \sum_{i=1}^{N} \sum_{j=i+1}^{N} E\left[R_{i}\right] E\left[R_{j}\right] \\
& =\sum_{i=1}^{N} p_{i}\left(1-p_{i}\right) Q_{i}+\left(\sum_{i=1}^{N} p_{i} Q_{i}\right)^{2} .
\end{aligned}
$$

Note that the expected total effective exhibits similar structure to the models incorporating stochastically proportional yield instead of binomial. Yet, the variance of the effective received is different in the sense that it is proportional to the order quantities in contrast to the stochastically proportional yield models in which the variance is proportional to the square of the order quantities. This characteristic of the binomial yield eliminates the need for diversification in order to obtain the risk pooling effect as shown in the rest of the work.

The inventory level of the described inventory system is a stochastic process. The process regenerates itself every time the inventory level hits zero. Thus, one can obtain the long-run average cost for the system using the renewal-reward analysis. The replenishment cycle is defined as the time elapsed between two consecutive instants at which the inventory level hits zero. The system orders from its suppliers once per cycle. Even though the problem setting is based on discrete inventory units, we treat the units as continuous variables for analytical convenience as is customary in the previous yield literature $[4,6,11]$.
The expected cycle length is

$E[$ Cycle Length $]=E[R] / D$

and the expected cycle cost is

$E[$ Cycle Cost $]=K\left(Q_{1}, \ldots, Q_{N}\right)+\sum_{i=1}^{N} c_{i} Q_{i}+\frac{h}{2 D} E\left[R^{2}\right]$

Finally, due to renewal-reward theorem, the long-run average cost for the system, denoted as $C R$, can be obtained from

$C R=\frac{E[\text { Cycle Cost }]}{E[\text { Cycle Length }]}$.

\section{Optimality results}

Define adjusted unit cost for supplier $i$ as

$A C_{i}=\frac{c_{i}}{p_{i}}+\frac{h}{2 D}\left(1-p_{i}\right)$.

Lemma 1. Let $i$ and $j$ be the indices for any two suppliers such that $Q_{i}, Q_{j}>0$, let $-\left(p_{i} / p_{j}\right) Q_{i}<\Delta<Q_{j}$, and let $K\left(Q_{1}, \ldots, Q_{N}\right)=K$. If the order quantity from supplier $j$ is decreased by $\Delta$, and the order quantity from supplier $i$ is increased by $\left(p_{j} / p_{i}\right) \Delta$, then the resulting increase in the long-run average cost is

$\left.C R\right|_{\substack{Q_{i}=q_{i}+\left(p_{j} / p_{i}\right) \Delta \\ Q_{j}=q_{j}-\Delta}}-\left.C R\right|_{\substack{Q_{i}=q_{i} \\ Q_{j}=q_{j}}}=\frac{D p_{j}}{E[R]}\left(A C_{i}-A C_{j}\right) \Delta$,

where the notation $\left.\operatorname{Exp}\right|_{\substack{Q_{i}=a \\ Q_{j}=b}}$ denotes the same expression as Exp when $Q_{i}$ is set to $a$ and $Q_{j}$ is set to $b$.

Proof. Using (4), we compute the expected cycle length under the new order quantities as

$$
\begin{aligned}
E & \text { Cycle Length }]\left.\right|_{\substack{Q_{i}=q_{i}+\left(p_{j} / p_{i}\right) \Delta \\
Q_{j}=q_{j}-\Delta}} \\
= & \frac{\sum_{l \neq i, j} p_{l} Q_{l}+p_{i}\left(q_{i}+\left(p_{j} / p_{i}\right) \Delta\right)+p_{j}\left(q_{j}-\Delta\right)}{D} \\
= & \frac{\sum_{l=1}^{N} p_{l} Q_{l}}{D}=\left.E[\text { Cycle Length }]\right|_{\substack{Q_{i}=q_{i} \\
Q_{j}=q_{j}}}
\end{aligned}
$$

and observe that the expected cycle length and the expected total effective received remain the same. Then using (5) and (9) we compute the change in the expected cycle cost as

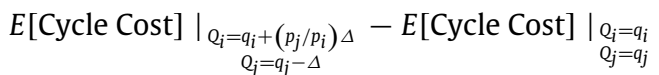

$$
\begin{aligned}
& =c_{i}\left(q_{i}+\left(p_{j} / p_{i}\right) \Delta-q_{i}\right)+c_{j}\left(q_{j}-\Delta-q_{j}\right) \\
& +\frac{h}{2 D}\left(p_{i}\left(1-p_{i}\right)\left(q_{i}+\left(p_{j} / p_{i}\right) \Delta-q_{i}\right)\right. \\
& \left.+p_{j}\left(1-p_{j}\right)\left(q_{j}-\Delta-q_{j}\right)\right) \\
& =c_{i}\left(p_{j} / p_{i}\right) \Delta-c_{j} \Delta+\frac{h}{2 D}\left(p_{j}\left(1-p_{i}\right) \Delta-p_{j}\left(1-p_{j}\right) \Delta\right) \\
& =p_{j}\left(\left(\frac{c_{i}}{p_{i}}+\frac{h}{2 D}\left(1-p_{i}\right)\right)-\left(\frac{c_{j}}{p_{j}}+\frac{h}{2 D}\left(1-p_{j}\right)\right)\right) \Delta \text {. }
\end{aligned}
$$

Finally by bringing together (6), (9) and (10) we obtain (8). The reader should note that this analysis is only valid when $-\left(p_{i} / p_{j}\right) Q_{i}<\Delta<Q_{j}$, since the order quantities always need to be positive. If one of the order quantities becomes zero, one would need to add the change in the ordering costs as well. 
When the ordering cost is constant, Lemma 1 suggests that it is better to order more from a supplier with a lower adjusted unit cost while reducing the order quantity from a supplier with a higher adjusted unit cost such that the total expected effective received is kept constant. Thus, by switching the order quantities in a proper fashion to a "cheaper" supplier we can always decrease the longrun average cost for the inventory system. If the adjusted unit costs of two suppliers are the same, no improvement (nor worsening) is possible by the kind of switch described.

It is easy to recognize that the first component of the adjusted unit cost, $c_{i} / p_{i}$, is the purchasing cost per good unit; whereas the second component, $(h / 2 D)\left(1-p_{i}\right)$, can be interpreted as the extra holding cost due to the variability caused by the unreliable nature of the ordered units. The latter disappears as the supplier becomes more reliable, i.e., as $p_{i}$ tends to one.

Theorem. Define $A C^{*}=\min _{1 \leq i \leq N} A C_{i}$ and $S^{*}=\left\{i \mid 1 \leq i \leq N, A C_{i}\right.$ $\left.=A C^{*}\right\}$. The following three results hold for three different ordering cost structures:

(i) Consider the setting when $K\left(Q_{1}, \ldots, Q_{N}\right)=K$. The ordering quantity vector $(s)\left(Q_{1}, \ldots, Q_{N}\right)$ satisfying the following two conditions are optimal:

1. For $i \notin S^{*}, Q_{i}=0$,

2. $\sum_{i \in S^{*}} p_{i} Q_{i}=\sqrt{\frac{2 K D}{h}}$.

(ii) Consider the setting when $K\left(Q_{1}, \ldots, Q_{N}\right)=K\left(\sum_{i=1}^{N}\right.$ $\left.1\left\{Q_{i}>0\right\}\right)$ where $K($.) is a positive monotonically increasing function and $1\left\{Q_{i}>0\right\}$ is the indicator function that returns 1 if $Q_{i}>0$ and returns 0 otherwise. The ordering quantity vector $(s)\left(Q_{1}, \ldots, Q_{N}\right)$ satisfying the following three conditions are optimal:

1. $\sum_{i=1}^{N} 1\left\{Q_{i}>0\right\}=1$,

2. for some $j \in S^{*}, Q_{j}=\frac{1}{p_{j}} \sqrt{\frac{2 K(.) D}{h}}$,

3. for $i \in\{1, \ldots, N\} \backslash\{j\}, Q_{i}=0$.

(iii) Consider the setting when $K\left(Q_{1}, \ldots, Q_{N}\right)=K+$ $\sum_{i=1}^{N} k_{i} 1\left\{Q_{i}>0\right\}$ where $K \geqslant 0$ and all $k_{i}>0,1 \leq i \leq N$. Define

$C R_{i}=\frac{\left(K+k_{i}\right) D}{p_{i} Q_{i}}+A C_{i} D+\frac{h p_{i} Q_{i}}{2}$,

$C R_{i}^{*}=\sqrt{2\left(K+k_{i}\right) D h}+A C_{i} D$,

and $\widetilde{S}^{*}=\left\{i \mid 1 \leq i \leq N, C R_{i}=C R_{i}^{*}\right\}$. The ordering quantity vector(s) $\left(Q_{1}, \ldots, Q_{N}\right)$ satisfying the following three conditions are optimal.

1. $\sum_{i=1}^{N} 1\left\{Q_{i}>0\right\}=1$,

2. for some $j \in \widetilde{S}^{*}, Q_{j}=\frac{1}{p_{j}} \sqrt{\frac{2\left(K+k_{j}\right) D}{h}}$,

3. for $i \in\{1, \ldots, N\} \backslash\{j\}, Q_{i}=0$.

Proof. (i) From Lemma 1, the long-run average cost can be continuously improved as order quantities are properly shifted (i.e., keeping the total expected effective received constant) from a supplier with higher unit adjusted cost to a supplier with lower unit adjusted cost until it hits zero. Thus, it is not optimal to order from any supplier not in $S^{*}$, consisting of the supplier(s) with the lowest unit adjusted cost.

Using (3) and (5), we now have

$$
\begin{aligned}
E[\text { Cycle Cost }]= & K+\sum_{i \in S^{*}} p_{i}\left(\frac{c_{i}}{p_{i}}+\frac{h}{2 D}\left(1-p_{i}\right)\right) Q_{i} \\
& +\frac{h}{2 D}\left(\sum_{i \in S^{*}} p_{i} Q_{i}\right)^{2} .
\end{aligned}
$$

Since the suppliers in $S *$ all have the same adjusted unit cost, $A C^{*}$, we have

$E[$ Cycle Cost $]=K+A C^{*} \sum_{i \in S^{*}} p_{i} Q_{i}+\frac{h}{2 D}\left(\sum_{i \in S^{*}} p_{i} Q_{i}\right)^{2}$.

Similarly (4) becomes

$E[$ Cycle Length $]=\frac{\sum_{i \in S^{*}} p_{i} Q_{i}}{D}$.

Hence,

$C R=\frac{K D}{\sum_{i \in S^{*}} p_{i} Q_{i}}+A C^{*} D+\frac{h}{2}\left(\sum_{i \in S^{*}} p_{i} Q_{i}\right)$.

Noting the canonical EOQ type cost structure, we obtain (11).

(ii) From Lemma 1 and the fact that the fixed ordering cost is monotonically increasing in the number of suppliers with positive order quantities, long-run average cost is improved by setting equal to zero the order quantities for suppliers not in $S^{*}$. For any given value of the expected total effective received $\left(\sum_{i \in S^{*}} p_{i} Q_{i}\right)$, long-run average cost is minimized when $\sum_{i=1}^{N} 1\left\{Q_{i}>0\right\}=1$, which implies operating with only one of the supplier(s) in $S^{*}$. The result follows from univariate optimization.

(iii) Consider the minimization of long-run average cost with respect to any two suppliers $i$ and $j$ such that $Q_{i}, Q_{j}>0$ and $A C_{i} \leq A C_{j}$, while keeping constant $Q_{l}, l \neq i, j$. If $k_{i}<k_{j}$, by Lemma 1 long-run average cost is improved by setting $Q_{j}=0$. If $k_{i} \geqslant k_{j}$, the best solution is obtained when either $Q_{j}=0$ or $Q_{i}=0$, since the interior points are suboptimal due to Lemma 1 . Hence, for any subset of two suppliers, it is optimal to work only with one of them. Proceeding in the same fashion with all possible supplier subsets of size 2, it is shown that it is optimal to work with a single supplier.

The average cost expression using single supplier $i$ can be obtained by modifying (17) as

$C R_{i}=\frac{\left(K+k_{i}\right) D}{p_{i} Q_{i}}+A C_{i} D+\frac{h p_{i} Q_{i}}{2}$.

The reader should note that this expression is just like the one in the canonical EOQ model with the exception of replacing $Q$ with $p_{i} Q_{i}$. Thereby, modifying the result for the EOQ model the optimal average cost with supplier $i$ yields

$C R_{i}^{*}=\sqrt{2\left(K+k_{i}\right) D h}+A C_{i} D$.

Thus, the optimal supplier to work with is found from

$i^{*}=\arg \min _{1 \leq i \leq N}\left\{C R_{i}^{*}\right\}$

and the optimal order quantity from this supplier is

$Q_{i^{*}}^{*}=\frac{1}{p_{i^{*}}} \sqrt{\frac{2\left(K+k_{i^{*}}\right) D}{h}}$.

Remark 1. If there are no supplier specific minor costs (as in (i) and (ii)), the suppliers with adjusted unit costs higher than the minimum available adjusted unit cost should never be used. In the presence of such costs (as in (iii)), it may be possible to exploit the trade-off between the ordering cost component and the remaining cost components, and work with the supplier that provides the best cost rate combination, but not necessarily has the lowest individual components. 
Remark 2. Consider the case when the fixed ordering cost is a constant. If the cardinality of the set $S^{*}$, i.e., the number of suppliers with the minimum adjusted unit cost, is greater than one, then the optimality conditions given in the above theorem do not yield a unique optimal solution. Infinitely many solutions lie over an optimal simplex which is the intersection of the positive quadrant and the hyperplane defined by a constant value of the expected total effective received. However, when we have the fixed ordering cost as strictly increasing with the number of suppliers used, i.e., $K_{n}>K_{m}$ given that $n>m$, then the set of optimal solutions is characterized by the vertices of the aforementioned simplex.

\section{Discussion}

A key observation from the analysis above is that if suppliers are subject to binomial yield uncertainty, to use a single supplier is always optimal. Although there may be other optimal configurations in cases where we are indifferent between suppliers, in practice an exact equality of adjusted unit prices is unlikely. This result constitutes a contrast to the conventional wisdom in the area of random yield. We provide this managerial finding in the following.

Corollary. (i) In the presence of constant fixed ordering cost, zero lead times and binomial yield, diversification does not bring additional cost benefit over using a single supplier.

(ii) When fixed ordering costs are monotonically increasing in the number of suppliers used or include supplier specific minor ordering costs, diversification is never optimal under binomial yields and zero lead times.

Diversification has been found to be beneficial due the risk pooling effects under yield structures investigated in the literature (e.g. stochastically proportional, all or nothing, random capacity). The underlying mathematical reason behind this phenomenon is that when we sum random variables, their variability, best manifested in their coefficient of variation, decreases.

In the case of binomial yield, the reason that diversification is not beneficial is that one can obtain variability reduction just by increasing the order quantities without resorting to additional suppliers. The critical fact to be observed is that the binomial distribution is itself a sum of Bernouilli distributions. As the order quantity from a supplier, i.e., number of trials in a binomial experiment increases, we sum up Bernouilli random variables and benefit from variability reduction. Thus, instead of obtaining risk pooling effect via supplier diversification, one can obtain it by simply augmenting the order from the "cheapest" supplier.

The three fixed ordering cost structures investigated herein are commonplace and correspond to realistic settings. For instance, the case in which the fixed ordering cost is an increasing function of the number of suppliers used, models the administrative burden due to the management effort in maintaining a large supplier base. Our finding is consistent with the recent efforts in many industries in reducing the supplier base. The fixed ordering cost structure with supplier specific minor costs is found when there are fixed components of freight costs in addition to administrative efforts, or when foreign suppliers are used with customs clearance costs.

\section{Extensions}

There are a number of possible extensions to the model herein. We discuss these next.

One of the assumptions of the model, which states that the purchasing cost is paid per item received irrespective of its quality, can be modified with a simple adjustment in the results. For example, if we assume that we only pay the purchasing cost, $c_{i}$, per "good" item received, all the derived results are valid once the adjusted unit cost (given in (7)) is redefined as

$A C_{i}=c_{i}+\frac{h}{2 D}\left(1-p_{i}\right)$.

A similar variation in the model is to allow the holding costs to depend on the purchasing price of the inventory. In this circumstance the inventory holding cost for one unit supplied from supplier $i$ per unit time can be expressed as $h_{i}=h+\hat{h} c_{i}$. If we assume that inventories originating from different suppliers are consumed simultaneously such that they all deplete at the same moment in time, i.e., when the inventory level drops to zero, the derived results are again valid given that the adjusted unit cost (given in (7)) is redefined as

$A C_{i}=\frac{c_{i}}{p_{i}}+\frac{h_{i}}{2 D}\left(1-p_{i}\right)$

A final variation that we would like to talk about is the incorporation of supplier capacities to the model. In the case that the capacities do not bind the optimal solution, the previous optimal solutions are still valid. Otherwise, we can only present a solution for the case that the ordering cost is constant irrespective of the number of suppliers used, i.e., $K_{n}=K$. In this case, one would have to sort the suppliers according to their adjusted unit costs and order from the "cheapest supplier" up to its capacity and then continue filling the supplier capacities until (11) is satisfied. If it cannot be satisfied even when all available supplier capacities are used then the process stops and ordering to full capacity is the optimal policy. If the most general cost structures along with capacity constraints are assumed then the problem becomes combinatorial in nature and there is little that can be done except trying all possible supplier combinations.

\section{References}

[1] R. Anupindi, R. Akella, Diversification under supply uncertainty, Management Science 39 (1993) 944-963.

[2] M. Barad, D. Braha, Control limits for multi-stage manufacturing processes with binomial yield (single and multiple production runs), The Journal of the Operational Research Society 47 (1996) 98-112.

[3] A. Beja, Optimal reject allowance with constant marginal production efficiency, Naval Research Logistics Quarterly 24 (1977) 21-33.

[4] A.S. Erdem, M.M. Fadiiloğlu, S. Özekici, An EOQ model with multiple suppliers and random capacity, Naval Research Logistics 53 (2006) 101-114.

[5] A.S. Erdem, S. Özekici, Inventory models with random yield in a random environment, International Journal of Production Economics 78 (2002) 239-253.

[6] Y. Gerchak, M. Parlar, Yield randomness, cost tradeoffs, and diversification in the EOQ model, Naval Research Logistics 37 (1990) 341-354.

[7] Y. Gerchak, A. Tripathy, K. Wang, Co-production models with random functionality yields, IIE Transactions 28 (1996) 391-403.

[8] A. Grosfeld-Nir, Y. Gerchak, Multiple lotsizing in production to order with random yields: Review of recent advances, Annals of Operations Research 126 (2004) 43-69.

[9] H. Gurnani, R. Akella, J. Lehoczky, Supply Management in assembly systems with random yield and random demand, IIE Transactions 32 (2000) 701-714.

[10] M.C. Gürbüz, An inventory model with two suppliers under yield uncertainty, Master's Thesis, Bilkent University, Ankara, Turkey, 2001

[11] J.B. Mazzola, W.F. McCoy, H.M. Wagner, Algorithms and Heuristics for Variable Yield Lot Sizing, Naval Research Logistics 34 (1987) 67-86.

[12] F.W. McFarlan, F. Young, Li \& Fung (A): Internet Issues, Harvard Business School, Boston, USA, 2005

[13] M. Parlar, D. Wang, Diversification under yield randomness in inventory models, European Journal of Operational Research 66 (1993) 52-64.

[14] M. Sepheri, E.A. Silver, C. New, A heuristic for multiple lotsizing for an order under variable yield, IIE Transactions 18 (1986) 63-69.

[15] Y. Wang, Y. Gerchak, Input control in a batch production system with lead times, due dates, and random yields, European Journal of Operational Research 126 (2000) 371-385.

[16] C.A. Yano, H.L. Lee, Lot sizing with random yields, Operations Research 126 (1995) 311-334. 